\title{
O APOIO MULTICRITÉRIO À DECISÃO COMO INSTRUMENTO DE GESTÃO DO CONHECIMENTO: UMA APLICAÇÃO À INDÚSTRIA DE REFINO DE PETRÓLEO
}

\author{
Carmen Lúcia de Almeida Meirelles* \\ Abastecimento-Refino / Tecnologia de Refino \\ Petrobras \\ Rio de Janeiro - RJ \\ carmenlam@petrobras.com.br \\ Luiz Flávio Autran Monteiro Gomes \\ Ibmec/RJ \\ Rio de Janeiro - RJ \\ autran@ibmecrj.br \\ * Corresponding author / autor para quem as correspondências devem ser encaminhadas \\ Recebido em 08/2008; aceito em 04/2009 após 1 revisão \\ Received August 2008; accepted April 2009 after one revision
}

\begin{abstract}
Resumo
Este artigo aborda o relacionamento entre a gestão do conhecimento e o uso de métodos multicritério para apoio à decisão. Como estudo de caso, apresenta uma aplicação da função de valor multiatributo à avaliação e seleção de tecnologias de refino de petróleo. O modelo de análise aqui empregado foi desenvolvido a partir de entrevistas com técnicos envolvidos na seleção de tecnologias, tendo sido validado por sua aplicação a um processo de seleção. Utilizou-se a técnica de swing weighting para a calibração da função de valor multiatributo, com uma análise de sensibilidade. A conclusão final do artigo é que os métodos multicritério podem se constituir em importantes instrumentos de gestão do conhecimento, desde que estejam previamente modelados em sistemas que simplifiquem a sua utilização, conferindo agilidade ao processo decisório. Recomenda-se a extensão de estudos de aplicação do apoio à decisão, como ferramenta de gestão do conhecimento, a outras áreas de aplicação.
\end{abstract}

Palavras-chave: apoio multicritério à decisão; gestão do conhecimento; indústria de petróleo.

\begin{abstract}
This article approaches the relationship between knowledge management and the use of multicriteria decision aiding methods. It presents a case study application of a multiattribute value function to the evaluation and selection of oil refining technologies. The analytical model utilized was developed based on interviews with professionals involved in the selection of technologies. That model was validated through its application to a selection process that was previously carried out. The swing weighting technique was used for the calibration of the multiattribute value function. A sensitivity analysis was also performed. The final conclusion from this article points out to the employment of multicriteria decision aiding methods as an important knowledge management tool. For this to be true these methods must be embedded in systems that would confer agility to decision making. It is recommended that additional experience must be gained with relating applications of multicriteria decision aiding to knowledge management processes.
\end{abstract}

Keywords: multicriteria decision aiding; knowledge management; petroleum industry. 


\section{Introdução}

A relação entre gestão do conhecimento e o Apoio Multicritério à Decisão foi conceituada por Zeleny (2005). Este autor apresentou então o quadro de referência amplo da Gestão de Sistemas Humanos (GSH) como a integração das três dimensões básicas dos negócios: conhecimento, gestão e sistemas. Em seguida, a partir do reconhecimento de que a GSH lida sempre com a multidimensionalidade, demonstrou Zeleny, no quarto capítulo de sua obra citada, a impossibilidade de se dissociar a GSH - e, por conseguinte, a gestão do conhecimento - e o uso dos métodos do Apoio Multicritério à Decisão. Zeleny (2005) mostrou, em essência, que inexiste gestão do conhecimento que passe ao largo do processo humano de tomada de decisão, sendo precisamente o Apoio Multicritério à Decisão o enfoque adequado ao tratamento de tal processo em sua multiplicidade de critérios e pontos de vista. Aquela mesma relação tinha sido anteriormente abordada na dissertação de mestrado de Santos (2003) e, posteriormente, na tese de doutorado de Campos (2005). A integração entre o Apoio Multicritério à Decisão e a gestão do conhecimento tem sido ainda tratada por autores como Trinkaus (2006); Kain, Kärrman \& Söderberg (2007); Feyzioğlu \& Büyüközkan (2007) e Rauscher, Schmoldt \& Vacik (2007).

Gomes \& Santos (2008) sugerem que, nas empresas, a importância das iniciativas que buscam montar estruturas que suportem analiticamente as decisões deve aumentar e propõem estudos de problemas de gestão do conhecimento que possam ser modelados quantitativamente, principalmente a simulação de ambientes fomentadores da produção do conhecimento.

Um processo de gestão do conhecimento pode ser visto a partir de duas óticas diferentes: uma centrada na informação e a outra centrada nos processos de aprendizagem. A visão centrada na informação considera a gestão do conhecimento como "a disciplina que promove uma abordagem integrada à identificação, gerenciamento e compartilhamento de todos os ativos de informação de uma empresa, incluindo bases de dados, documentos, políticas e procedimentos, assim como competências e experiências não claramente explicitadas, residentes nos indivíduos". A visão centrada nos processos de aprendizagem tem como base a premissa de que não importa o quão adequadamente a informação seja gerenciada, se a mesma não pode ser bem compreendida pelos indivíduos na organização ou convertida em ação ou na habilidade para "fazer" (Morey, Mayburg \& Thuraisingham, 2002).

Nonaka \& Takeuchi (1995) apresentam os conceitos de conhecimento tácito e conhecimento explícito. Por conhecimento tácito entende-se o conhecimento pessoal, específico ao contexto e, por isso, difícil de ser formulado e comunicado; o conhecimento explícito ou codificado refere-se ao conhecimento transmissível em linguagem formal e sistemática. Com base nesses conceitos, diversos autores sustentam que a gestão do conhecimento deve prover métodos para gerenciar tanto o conhecimento tácito como o explícito, de forma que estes possam ser usados para resolver problemas, explorar oportunidades ou tomar decisões que melhorem a performance (Rauscher, Schmoldt \& Vacik, op.cit.).

Uma vez estabelecida a relação entre gestão do conhecimento e Apoio Multicritério à Decisão, fica evidente que a adoção dos métodos analíticos de apoio à decisão como instrumento de gestão do conhecimento contempla as diversas óticas supracitadas. $\mathrm{O}$ processo de identificação dos parâmetros importantes e a modelagem do problema permitem a conversão dos conhecimentos tácitos dos especialistas em conhecimentos explícitos e a integração desses diversos conhecimentos especializados em prol de um objetivo comum que é a tomada de decisão. Por outro lado, a adoção de preceitos lógicos e explícitos permite o questionamento e reavaliação do processo decisório e a consequente aprendizagem organizacional. 
Assim sendo, a proposta desse artigo é demonstrar como um método analítico de apoio à decisão pode se constituir em um instrumento de gestão do conhecimento através da aplicação do método da função de valor multiatributo à comparação e seleção de tecnologias de processos de refino de petróleo. Por esta razão, na seção 2 deste artigo apresentam-se os fundamentos metodológicos da análise multicritério realizada, ao passo que, na sua seção 3 , apresenta-se a aplicação dos mesmos.

\section{A teoria da decisão}

\subsection{Formulação do problema}

Decisões permeiam todas as atividades humanas, seja em nível pessoal ou em nível organizacional, e muitas dessas decisões são tomadas de maneira informal ou intuitiva. No entanto, ao longo dos tempos, a necessidade de melhores decisões levou à busca de abordagens sistemáticas e estruturadas que conduzissem a um processo decisório mais satisfatório.

O objetivo principal de uma ferramenta de apoio à decisão é aprimorar sua racionalidade, ou seja, aumentar a perspectiva de que uma escolha conduza a um resultado satisfatório. Escolha racional pode ser definida como sendo aquela que se baseia em tudo que o decisor sabe, julga e sente, satisfazendo suas preferências de forma eficaz e lógica. A racionalidade em uma decisão melhora sua qualidade na medida em que se baseia em todo o conhecimento e expertise disponíveis, além de tornar transparentes as motivações subjacentes (Brown, 2005).

No campo do apoio à tomada de decisão na presença de múltiplos critérios, a formulação do problema tem sido tratada por meio da denominada estruturação do problema (Montibeller Neto et al., 2008; Ensslin, Montibeller Neto \& Noronha, 2001; Rosenhead \& Mingers, 2008; Belton, Ackermann \& Sheperd, 1997). No caso específico do estudo de caso aqui apresentado, a estruturação do problema se deu a partir de discussões de técnicos de diversas especialidades (projeto, operação, manutenção e meio ambiente); a modelagem procurou representar os requisitos técnicos e econômicos fundamentais que devem ser atendidos do ponto de vista dos diversos especialistas, chegando-se a uma compreensão consensualmente aceita do problema. Esta compreensão, que inclui a explicitação dos objetivos decomponíveis nos critérios de decisão, bem como a identificação das alternativas viáveis, é o que se entende por um modelo requisito para o problema (Philips, 1984, 1990).

Neste artigo aplica-se um método analítico do Apoio Multicritério à Decisão, a função de valor multiatributo, à seleção de tecnologias de processos de refino de petróleo, a partir de uma modelagem que permita a utilização do método de forma interativa. Para uma apresentação das principais características de um conjunto amplo de métodos analíticos do Apoio Multicritério à Decisão consulte-se Figueira, Greco \& Ehrgott (2005).

\subsection{O enfoque multicritério}

\subsubsection{A teoria da utilidade e sua extensão multiatributo}

Em 1789, Daniel Bernoulli apresenta o conceito de utilidade como unidade de medida de preferência. Bentham definiu utilidade como "aquela propriedade em qualquer objeto, pela qual ele tende a produzir benefício, vantagem, prazer, bem ou infelicidade... ou... evitar a ocorrência de dano, dor, mal ou infelicidade" (Bentham, 1789). A formalização da teoria da utilidade efetuada por Von Neumann \& Morgenstern (1947), posteriormente refinada por

Pesquisa Operacional, v.29, n.2, p.451-470, Maio a Agosto de 2009 
Fishburn (1970) e Keeney \& Raiffa (1976), serviu de base para a formulação de uma teoria de preferência para escolhas envolvendo riscos, ou seja, "loterias ou jogos com saídas que dependem de um conjunto de eventos mutuamente exclusivos e exaustivos" (Dyer, 2005, p.271). Keeney \& Raiffa (1976) estenderam os conceitos da teoria da utilidade para o auxílio a problemas decisórios, nos quais cada alternativa pudesse ser descrita por uma lista de atributos. Os autores propuseram a construção de uma função matemática, capaz de agregar as informações dos múltiplos atributos de forma que, a cada alternativa, pudesse ser associada uma medida de valor. Isto torna possível elaborar ordens de preferências entre as alternativas. Esses autores definiram uma função de representação de preferência, sob condições de certeza, como "função de valor" e uma função de representação de preferência, sob condições de risco, como "função de utilidade". A função de utilidade multiatributo pode ter diversas formas matemáticas e sua aplicação, como instrumento de apoio à decisão, requer o atendimento a alguns requisitos. Um desses requisitos é a construção de um modelo que permita ao analista comparar as alternativas, com base em um conjunto de critérios, ou seja, um modelo que permita estabelecer relações de preferência entre alternativas.

Bouyssou $(1990$, p.59) ressalta que o sucesso de um sistema de apoio à decisão depende crucialmente da maneira como a família de critérios é construída, devendo levar em conta diversos pontos de vista que "representam os diferentes eixos ao longo dos quais os diversos atores do processo decisório justificam, transformam e questionam suas preferências". A família de critérios deve ser compreendida e admitida por todos os envolvidos no processo decisório, levando em conta as respectivas culturas. A forma de organização dos critérios mais utilizada é a construção de uma hierarquia em forma de uma árvore, na qual se decompõe cada critério progressivamente, partindo do critério mais alto para aqueles de nível mais baixo. A construção de uma família de critérios deve satisfazer a algumas condições técnicas para que possa ser adequadamente aplicada.

O método da teoria da utilidade multiatributo (também designado por MAUT Multiattribute Utility Theory) considera que, para cada alternativa a ser avaliada, existe um conjunto de atributos que a caracteriza e, para cada um dos atributos, existe uma função de utilidade específica que associa uma medida de valor a esse atributo. A função de utilidade multiatributo é a agregação das diferentes funções de utilidade de cada atributo, ponderadas conforme o grau de importância do atributo.

Dyer (2005) apresenta uma revisão da teoria da utilidade multiatributo. Para esse autor, MAUT seria um acrônimo mais geral, abrangendo diversos modelos multiatributo de escolhas. O mesmo autor argumenta que, apesar de muitos acadêmicos considerarem a teoria de utilidade multiatributo de Keeney \& Raiffa (1976) como sinônimo da teoria da preferência multiatributo, essa teoria não é apropriada para decisões envolvendo múltiplos objetivos, quando o risco não é um fator a ser considerado, tendo em vista que os axiomas apropriados para escolhas de risco não têm que ser satisfeitos para utilização de modelos de preferência multiatributos que não envolvam riscos. Belton \& Stewart (2002) adotam a nomenclatura "teoria de valor multiatributo" (ou MAVT - Multiattribute Value Theory) e consideram que a teoria de utilidade pode ser vista como uma extensão da teoria de valor aplicada a situações de incerteza. Dyer (2005), por sua vez, adota a mesma definição proposta por Keeney \& Raiffa (1976) e Belton \& Stewart (2002), referindo-se a uma função de representação de preferência sob certeza, como "função de valor" e a uma função de representação sob risco, como "função de utilidade".

As teorias de preferência multiatributo para condições de certeza são baseadas em comparações ordinais de alternativas ou em estimativas da força de preferência entre pares 
de alternativas. Essas teorias são baseadas em rigorosos axiomas que caracterizam o comportamento de escolha dos indivíduos e são essenciais para o estabelecimento de funções de representação de preferências, constituindo-se na base lógica para a análise quantitativa de preferências (Dyer, 2005).

\subsubsection{A Função de valor multiatributo}

A abordagem mais comum para avaliação de alternativas multiatributo é usar uma representação aditiva, ou seja:

$$
\mathrm{v}\left(\mathrm{x}_{1}, \ldots, \mathrm{x}_{\mathrm{n}}\right)=\sum_{\mathrm{i}=1}^{\mathrm{n}} \mathrm{v}_{\mathrm{i}}\left(\mathrm{x}_{\mathrm{i}}\right)
$$

Onde $\mathrm{v}_{\mathrm{i}}$ são funções de valor do atributo único $\mathrm{x}_{\mathrm{i}}$.

A condição chave para utilização da representação aditiva é que haja mútua independência de preferência entre os atributos $\mathrm{X}_{\mathrm{i}}$. Os atributos $\mathrm{X}_{\mathrm{i}}$ e $\mathrm{X}_{\mathrm{j}}$ são preferencialmente independentes se os tradeoffs entre $\mathrm{X}_{\mathrm{i}}$ e $\mathrm{X}_{\mathrm{j}}$ são independentes de todos os outros atributos. Mútua independência de preferência requer que a independência de preferência se sustente para todos os pares $\mathrm{X}_{\mathrm{i}}$ e $\mathrm{X}_{\mathrm{j}}$, ou seja, as curvas de indiferença para quaisquer pares de atributos devem ser inalteradas por níveis fixos dos atributos remanescentes. Nos casos em que uma representação aditiva não possa ser utilizada, uma forma multiplicativa deve ser considerada. Nesse caso, outras condições mais rigorosas devem ser satisfeitas (Keeney \& Raiffa, 1976; Clemen \& Reilly, 2001; Dyer, 2005).

O conceito de compensação em estruturas de preferência multiatributo se refere à existência de tradeoffs, ou seja, à possibilidade de contrabalançar uma desvantagem com relação a algum atributo, com uma vantagem suficientemente grande em outro atributo. Assim, uma relação de preferência é não compensatória se não há ocorrência de tradeoffs e compensatória, em caso contrário (Bouyssou, 1990). Bouyssou (1990, p.153) define ainda o conceito de convenção de agregação, como "a maneira pela qual um processo analítico transforma a informação a fim de chegar a uma ordenação de preferências". Assim, uma convenção de agregação é minimamente compensatória se, para algum conjunto $X$, a ordenação de preferências pode produzir uma relação de preferência na qual I minimamente compensa J, para algum I e J, e, não compensatória, se ocorrer o contrário. Dentro desse enfoque, a convenção subjacente à representação aditiva de uma função de valor é clara e minimamente compensatória.

Apresentações dos fundamentos da teoria da utilidade multiatributo e de suas representações analíticas podem ainda ser encontradas em Belton \& Stewart (2002); Souza (2002); e Gomes, Gomes \& Almeida (2006, p.159-206). O artigo de Wallenius et al. (2008) mostra os mais recentes avanços e sugere desenvolvimentos futuros da teoria da utilidade multiatributo.

\section{Estudo de Caso}

\subsection{Contexto: A seleção de tecnologias de refino de petróleo}

O petróleo extraído do poço não tem aplicação direta; a sua utilização ocorre por meio de seus derivados. O processo de conversão em derivados próprios aos diversos consumos é denominado refino de petróleo. Os derivados de petróleo mais conhecidos são: gás liquefeito de petróleo, gasolinas, naftas, óleo diesel, querosenes de aviação e de iluminação, óleos combustíveis, asfaltos, lubrificantes, combustíveis marítimos, solventes, parafinas e coque de petróleo.

Pesquisa Operacional, v.29, n.2, p.451-470, Maio a Agosto de 2009 
As parcelas de cada produto obtido no refino dependem da qualidade do petróleo que está sendo processado e da estrutura da refinaria, ou seja, de suas unidades de processo. Os principais processos de refino normalmente empregados em refinarias são: dessalgação, destilação atmosférica e a vácuo, craqueamento catalítico, hidrocraqueamento, hidrotratamento, reforma, coqueamento, alquilação, isomerização, além de tratamentos auxiliares que visam a adequação da qualidade de produtos e dos efluentes a serem descartados (Refining Processes Handbook, 2008).

A qualidade do petróleo varia com o tempo e com o local da exploração, de forma que descobertas de novos campos de produção implicam na necessidade de adequação do parque de refino, com adaptação das unidades de processo existentes, e/ou a inclusão de novas unidades. Também demandas de mercado e mudanças na legislação podem resultar na necessidade de novas unidades de processo.

Para cada um dos processos de refino existem alguns licenciadores, cujas tecnologias podem diferir em diversos aspectos. A seleção de tecnologias de refino se constitui em uma tarefa bastante complexa, pois requer a integração de conhecimentos especializados, envolvendo perspectivas diversas e muitas vezes com objetivos conflitantes. É um processo decisório que envolve fortemente questões técnicas além das questões econômicas.

A avaliação de tecnologias desenvolvidas por diversos licenciadores é feita por grupos multidisciplinares de especialistas das áreas de operação, projeto, manutenção, meio ambiente e segurança; esses grupos variam conforme a tecnologia de processo a ser avaliada e o local a ser implantada a nova tecnologia. Trata-se, portanto, de um processo decisório que envolve altos valores financeiros e requer conhecimentos especializados; daí a importância da qualidade da decisão que, em última análise, é decorrente da adequada gestão do conhecimento tecnológico, em suas vertentes de criação, explicitação, transmissão e integração.

O estudo de caso em questão teve como base o processo de avaliação de três tecnologias de determinado processo de refino e a seleção daquela que melhor atendeu aos objetivos propostos.

\subsection{Metodologia}

O método multicritério de apoio à decisão escolhido para modelar o problema da seleção de tecnologias de refino de petróleo foi à função de valor multiatributo.

A escolha da função de valor multiatributo como método multicritério para a modelagem do problema foi decorrente do fato de ser este o método que apresenta uma base axiomática sólida, no sentido de procurar descrever o comportamento dos indivíduos em seu processo decisório. Associado a isso, foi o método que se mostrou mais adequado às características do problema, na medida em que permitiu representar os diferentes eixos ao longo dos quais os técnicos que executam a tarefa de seleção de tecnologias justificam suas preferências. Por ser um método compensatório permite explicitar os tradeoffs e tratá-los de forma adequada.

A modelagem foi desenvolvida com base no pressuposto que cada tecnologia deve atender a um conjunto de objetivos fundamentais, estabelecidos por técnicos de diversas especialidades. Cada um dos objetivos fundamentais pode ser decomposto em um conjunto de atributos organizados hierarquicamente. Aos objetivos fundamentais, assim como aos atributos de níveis inferiores na hierarquia, são atribuídos pesos conforme o grau de importância conferido pelos técnicos. Por fim, calcula-se a função de valor global que permitirá selecionar a tecnologia que apresentar o maior valor para o conjunto de objetivos estabelecidos. 
A modelagem do sistema consistiu nas seguintes etapas:

1. Estabelecimento dos objetivos fundamentais

2. Definição e organização dos atributos em uma árvore

3. Estabelecimento de escalas de medição para os atributos

4. Avaliação da ocorrência de interação entre os atributos

5. Atribuição de pesos aos atributos

6. Cálculo da função de valor

7. Escolha da alternativa com maior função de valor global

\subsubsection{Construção da árvore dos atributos}

A partir de entrevistas com especialistas das áreas de projeto, operação, manutenção e meio ambiente foram estabelecidos os objetivos fundamentais a serem atingidos por cada uma das tecnologias avaliadas.

Para cada objetivo fundamental, foram definidos atributos capazes de expressar os objetivos fundamentais de forma mensurável. Os atributos que por si só podiam ser expressos por valores numéricos tiveram esses valores adotados como critério de medição. Para os atributos que não eram diretamente quantificáveis, foram estabelecidas escalas que permitissem a conversão de variáveis qualitativas em quantitativas. A Tabela 1 apresenta a árvore resultante da sistemática descrita.

Tabela 1 - Árvore Hierárquica $\left(\mathrm{H}_{2} \mathrm{~S}\right.$ : Sulfeto de Hidrogênio, $\mathrm{NH}_{3}$ : Amônia, $\mathrm{CO}_{2}$ : Dióxido de Carbono, $\mathrm{SO}_{\mathrm{x}}$ : Óxidos de Enxofre, $\mathrm{NO}_{\mathrm{x}}$ : Óxidos de Nitrogênio, COV: Compostos Orgânicos Voláteis).

\begin{tabular}{|c|c|c|c|}
\hline $\begin{array}{c}\text { Objetivos } \\
\text { Fundamentais }\end{array}$ & $\begin{array}{l}\text { Atributos de } \\
\text { primeiro nível }\end{array}$ & $\begin{array}{l}\text { Atributos de } \\
\text { segundo nível }\end{array}$ & $\begin{array}{l}\text { Atributos de } \\
\text { terceiro nível }\end{array}$ \\
\hline $\begin{array}{l}\text { Maximizar o valor } \\
\text { presente líquido }\end{array}$ & $\begin{array}{l}\text { Valor presente } \\
\text { líquido }\end{array}$ & & \\
\hline \multirow{18}{*}{$\begin{array}{l}\text { Maximizar } \\
\text { facilidade de } \\
\text { manutenção }\end{array}$} & \multirow{18}{*}{$\begin{array}{l}\text { Facilidade de } \\
\text { manutenção }\end{array}$} & \multirow{9}{*}{$\begin{array}{l}\text { Dimensão dos } \\
\text { equipamentos }\end{array}$} & Fornos \\
\hline & & & Reatores e vasos \\
\hline & & & Torres e bandejas \\
\hline & & & Compressores \\
\hline & & & Filtros \\
\hline & & & Permutadores de calor \\
\hline & & & Bombas \\
\hline & & & Vasos \\
\hline & & & Acionadores \\
\hline & & \multirow{9}{*}{$\begin{array}{c}\text { Facilidades } \\
\text { em geral }\end{array}$} & Redundância de equipamentos \\
\hline & & & \begin{tabular}{|l|} 
Disposição horizontal \\
\end{tabular} \\
\hline & & & \begin{tabular}{|l|} 
Intercambiabilidade de peças \\
\end{tabular} \\
\hline & & & \begin{tabular}{|c|}
$\begin{array}{c}\text { Sistemas liberáveis sem parada } \\
\text { geral }\end{array}$ \\
\end{tabular} \\
\hline & & & \begin{tabular}{|c|} 
Diversas entradas e saídas em \\
equipamentos
\end{tabular} \\
\hline & & & Emprego de materiais comuns \\
\hline & & & Sistemas na forma de skids \\
\hline & & & Dispositivos de fácil abertura \\
\hline & & & Área ocupada \\
\hline
\end{tabular}




\begin{tabular}{|c|c|c|c|}
\hline \multirow{10}{*}{$\begin{array}{l}\text { Minimizar } \\
\text { riscos ambientais }\end{array}$} & \multirow{10}{*}{$\begin{array}{l}\text { Riscos } \\
\text { ambientais }\end{array}$} & \multirow{4}{*}{ Emissões líquidas } & Outros \\
\hline & & & $\mathrm{H}_{2} \mathrm{~S}$ \\
\hline & & & $\mathrm{NH}_{3}$ \\
\hline & & & Hidrocarbonetos \\
\hline & & \multirow{5}{*}{ Emissões gasosas } & $\mathrm{CO}_{2}$ \\
\hline & & & $\mathrm{SO}_{\mathrm{x}}$ \\
\hline & & & $\mathrm{NO}_{\mathrm{x}}$ \\
\hline & & & $\mathrm{COV}$ \\
\hline & & & Particulados \\
\hline & & Descarte de sólidos & Resíduos sólidos \\
\hline \multirow{2}{*}{$\begin{array}{l}\text { Minimizar } \\
\text { riscos tecnológicos }\end{array}$} & \multirow{2}{*}{$\begin{array}{l}\text { Riscos } \\
\text { tecnológicos }\end{array}$} & $\begin{array}{l}\text { Número de plantas em } \\
\text { construção/projeto }\end{array}$ & \\
\hline & & $\begin{array}{l}\text { Número de plantas em } \\
\text { operação }\end{array}$ & \\
\hline $\begin{array}{c}\text { Minimizar } \\
\text { consumo de energia }\end{array}$ & $\begin{array}{l}\text { Consumo de } \\
\text { energia }\end{array}$ & Índice de energia & \\
\hline $\begin{array}{l}\text { Minimizar } \\
\text { consumo de água }\end{array}$ & $\begin{array}{l}\text { Consumo de } \\
\text { água }\end{array}$ & $\begin{array}{l}\text { Volume de água } \\
\text { consumido }\end{array}$ & \\
\hline
\end{tabular}

\subsubsection{Determinação das escalas de medição}

Para o atributo de primeiro nível hierárquico "Valor presente líquido", foi adotada uma escala numérica monetária. $\mathrm{O}$ atributo de primeiro nível "Facilidade de manutenção" foi subdividido em dois atributos de segundo nível: "Dimensão dos equipamentos" e "Facilidades em geral". Para o atributo "Dimensão dos equipamentos", foi adotada uma escala cardinal, com valores expressos em toneladas.

Para o atributo "Facilidades em geral", foi adotada uma escala ordinal convertida em numérica, na qual o melhor resultado deveria ser expresso por uma maior pontuação; ou seja, as três tecnologias analisadas foram ordenadas considerando o seu desempenho com relação a cada um dos atributos de terceiro nível que constituem o atributo "Facilidades em geral". A tecnologia que melhor atendeu ao atributo recebeu pontuação 3 e a que pior atendeu recebeu pontuação 1. À tecnologia que obteve maior pontuação global, foi atribuído valor 1 e à que obteve menor pontuação foi atribuído o valor 0 para o atributo "Facilidades em geral". Tendo sido definidos os dois extremos da escala, o valor para a terceira tecnologia foi obtido por interpolação direta.

O atributo de primeiro nível "Riscos ambientais" foi subdividido em três atributos de segundo nível: "Emissões líquidas", "Emissões gasosas" e "Descarte de sólidos"; para esses atributos foi adotada uma escala numérica ponderal, expressa em toneladas/mês.

O atributo de primeiro nível "Riscos tecnológicos" foi subdividido em dois atributos de segundo nível: "Número de plantas em operação" e "Número de plantas em construção ou projeto", expressos por escala numérica. Esses atributos podem ser relacionados à "idade" da tecnologia, tendo em vista que maior número de plantas em operação é um indicativo de tecnologias mais maduras ou consolidadas e que, portanto implicam em menores riscos tecnológicos. 
O atributo de primeiro nível "Consumo energia" foi expresso pelo "Índice de energia”, valor numérico adimensional, normalmente utilizado para a avaliação do grau de otimização energética do processo.

O atributo de primeiro nível "Consumo de água" foi representado por uma escala numérica, expressa em metros cúbicos/hora.

A Tabela 2 apresenta um resumo das escalas adotadas para os diferentes atributos.

Tabela 2 - Escalas de medição.

\begin{tabular}{|c|c|c|c|}
\hline Objetivo Fundamental & Atributo & \multicolumn{2}{|c|}{ Escala } \\
\hline $\begin{array}{c}\text { Maximizar valor presente } \\
\text { líquido }\end{array}$ & $\begin{array}{c}\text { Valor presente } \\
\text { líquido }\end{array}$ & Numérica monetária & Milhões de dólares \\
\hline $\begin{array}{c}\text { Maximizar facilidade de } \\
\text { manutenção }\end{array}$ & $\begin{array}{c}\text { Dimensão dos } \\
\text { equipamentos }\end{array}$ & Cardinal & Toneladas \\
\cline { 2 - 4 } & Facilidades em geral & $\begin{array}{c}\text { Ordinal, convertida } \\
\text { em numérica. } \\
\text { maior pontuação }\end{array}$ & Adimensional \\
\hline $\begin{array}{c}\text { Minimizar riscos } \\
\text { ambientais }\end{array}$ & $\begin{array}{c}\text { Emissões líquidas } \\
\text { Emissões gasosas } \\
\text { Descarte de sólidos }\end{array}$ & $\begin{array}{c}\text { Numérica ponderal } \\
\text { Riscos tecnológicos }\end{array}$ & Noneladas/mês \\
\hline $\begin{array}{c}\text { Minimizar riscos } \\
\text { tecnológicos }\end{array}$ & Índice de energia & Numérica & Adimensional \\
\hline $\begin{array}{c}\text { Minimizar consumo } \\
\text { de energia }\end{array}$ & Volume consumido & Numérica & m ${ }^{3} /$ hora \\
\hline $\begin{array}{c}\text { Minimizar consumonal } \\
\text { de água }\end{array}$ & Numsional \\
\hline
\end{tabular}

\subsubsection{Avaliação da ocorrência de interação entre os atributos}

A avaliação da ocorrência de interação entre os atributos de primeiro nível foi feita a partir da metodologia sugerida por Keeney \& Raiffa (1976), que permite verificar a existência de influência de determinado atributo sobre os demais no tocante à escolha (Clemen \& Reilly, 2001).

Foi perguntado aos técnicos das diversas especialidades se a fixação de cada um dos atributos de primeiro nível, em valores diversos, ocasionaria alteração na escolha com relação aos demais atributos. Para todos os atributos foram obtidas respostas negativas, o que caracterizou a inexistência de interação entre os atributos. 
Tabela 3 - Interação entre os atributos de primeiro nível hierárquico.

\begin{tabular}{|c|c|c|}
\hline $\begin{array}{l}\text { Atributos de primeiro nível } \\
\text { fixados }\end{array}$ & $\begin{array}{l}\text { Atributos de primeiro nível } \\
\text { de comparação }\end{array}$ & $\begin{array}{c}\text { Escolha com relação ao } \\
\text { atributo fixado }\end{array}$ \\
\hline \multirow{4}{*}{ Valor presente líquido } & Facilidade de manutenção & Não se altera \\
\hline & Riscos ambientais & Não se altera \\
\hline & Consumo de energia & Não se altera \\
\hline & Consumo de água & Não se altera \\
\hline \multirow{4}{*}{ Facilidade de manutenção } & Valor presente líquido & Não se altera \\
\hline & Riscos ambientais & Não se altera \\
\hline & Consumo de energia & Não se altera \\
\hline & Consumo de água & Não se altera \\
\hline \multirow{4}{*}{ Riscos ambientais } & Valor presente líquido & Não se altera \\
\hline & Facilidade de manutenção & Não se altera \\
\hline & Consumo de energia & Não se altera \\
\hline & Consumo de água & Não se altera \\
\hline \multirow{5}{*}{ Riscos tecnológicos } & Valor presente líquido & Não se altera \\
\hline & Facilidade de manutenção & Não se altera \\
\hline & Riscos ambientais & Não se altera \\
\hline & Consumo de energia & Não se altera \\
\hline & Consumo de água & Não se altera \\
\hline \multirow{4}{*}{ Consumo de energia } & Valor presente líquido & Não se altera \\
\hline & Facilidade de manutenção & Não se altera \\
\hline & Riscos ambientais & Não se altera \\
\hline & Consumo de água & Não se altera \\
\hline \multirow{4}{*}{ Consumo de água } & Valor presente líquido & Não se altera \\
\hline & Facilidade de manutenção & Não se altera \\
\hline & Riscos ambientais & Não se altera \\
\hline & Consumo de energia & Não se altera \\
\hline
\end{tabular}

\subsubsection{Determinação dos pesos dos atributos}

\subsubsection{Pesos dos atributos de primeiro nível hierárquico}

Para a determinação dos pesos dos atributos de primeiro nível hierárquico foi utilizado o método swing weighting, que "consiste na comparação individual dos atributos diretamente imaginando um resultado hipotético" (Clemen \& Reilly, 2001, p.547).

O primeiro passo na aplicação do método consistiu na elaboração de uma tabela, em cuja primeira linha o pior resultado possível foi inserido. Em cada uma das linhas seguintes, um determinado atributo teve seu resultado substituído do pior para o melhor possível. 
O passo seguinte consistiu na solicitação aos técnicos especialistas que fizessem a ordenação dos resultados hipotéticos, tendo sido atribuída última posição ao pior resultado possível, ou seja, o pior resultado em todos os atributos. Em seguida, os outros resultados foram comparados de forma a se determinar a primeira posição e as posições seguintes na ordenação.

Na sequência, foi associada uma pontuação aos diversos resultados; ao último colocado na ordenação foi atribuído o valor 0 (zero) e ao melhor o valor 100 (cem). As pontuações para os outros resultados deveriam se situar entre 0 e 100 , tendo sido obtidas por comparações entre elas e entre os dois extremos.

A atribuição de pesos feita pelos técnicos levou em conta diversos fatores, tais como, a tecnologia de processo avaliada, o objetivo estratégico e a localização da planta. Para cada conjunto de tecnologias a serem avaliadas, caberá ao grupo multidisciplinar avaliar e chegar a um consenso com relação aos pesos a serem adotados. Os pesos obtidos para os atributos de primeiro nível hierárquico estão listados na Tabela 4.

Tabela 4 - Pesos dos atributos de primeiro nível hierárquico.

\begin{tabular}{|c|c|c|c|c|}
\hline Avaliação & Atributo & Ordenação & Pontuação & Peso \\
\hline $\begin{array}{c}\text { Benchmark } \\
\text { (pior resultado) }\end{array}$ & $\begin{array}{l}\text { Valor presente líquido } \\
\text { Riscos ambientais } \\
\text { Facilidade manutenção } \\
\text { Riscos tecnológicos } \\
\text { Consumo de energia } \\
\text { Consumo de água }\end{array}$ & 7 & 0 & 0,00 \\
\hline Melhor & $\begin{array}{l}\text { Valor presente líquido } \\
\text { Riscos ambientais } \\
\text { Facilidade manutenção } \\
\text { Riscos tecnológicos } \\
\text { Consumo de energia } \\
\text { Consumo de água }\end{array}$ & 1 & 100 & 0,27 \\
\hline Melhor & $\begin{array}{l}\text { Valor presente líquido } \\
\text { Riscos ambientais } \\
\text { Facilidade manutenção } \\
\text { Riscos tecnológicos } \\
\text { Consumo de energia } \\
\text { Consumo de água }\end{array}$ & 3 & 70 & 0,19 \\
\hline Melhor & $\begin{array}{l}\text { Valor presente líquido } \\
\text { Riscos ambientais } \\
\text { Facilidade manutenção } \\
\text { Riscos tecnológicos } \\
\text { Consumo de energia } \\
\text { Consumo de água }\end{array}$ & 4 & 50 & 0,13 \\
\hline Melhor & $\begin{array}{l}\text { Valor presente líquido } \\
\text { Riscos ambientais } \\
\text { Facilidade manutenção } \\
\text { Riscos tecnológicos } \\
\text { Consumo de energia } \\
\text { Consumo de água }\end{array}$ & 2 & 80 & 0,22 \\
\hline
\end{tabular}




\begin{tabular}{|c|c|c|c|c|}
\hline Melhor & $\begin{array}{c}\text { Valor presente líquido } \\
\text { Riscos ambientais } \\
\text { Facilidade manutenção } \\
\text { Riscos tecnológicos } \\
\text { Consumo de energia } \\
\text { Consumo de água }\end{array}$ & 5 & 40 & 0,11 \\
\hline Melhor & $\begin{array}{c}\text { Valor presente líquido } \\
\text { Riscos ambientais } \\
\text { Facilidade manutenção } \\
\text { Riscos tecnológicos } \\
\text { Consumo de energia } \\
\text { Consumo de água }\end{array}$ & 6 & 30 & 0,08 \\
\hline & Total & 370 & 1 \\
\hline
\end{tabular}

\subsubsection{Pesos dos atributos de segundo e terceiro níveis hierárquicos}

A atribuição de pesos aos atributos do segundo e terceiro níveis hierárquicos foi feita de modo direto pelos técnicos especialistas, conforme suas avaliações com relação ao grau de importância daqueles atributos. A análise levou em conta fatores técnicos e o conhecimento tácito daqueles especialistas.

Os atributos de segundo e terceiro níveis que compõem o atributo de primeiro nível "Facilidade manutenção" foi o que apresentou maior dificuldade de modelagem devido à necessidade de representar de forma mensurável o conhecimento tácito dos técnicos que realizam a montagem e manutenção dos equipamentos nas plantas industriais. Esses especialistas foram questionados sobre os cuidados especiais que deveriam ser tomados durante a fase de projeto de forma a facilitar as atividades posteriores de manutenção.

Da mesma forma, os pesos dos atributos de segundo e terceiro níveis que constituem o atributo de primeiro nível "Riscos ambientais" foram atribuídos pelos técnicos especialistas em meio ambiente, que consideraram que as emissões sólidas, líquidas e gasosas deveriam ser consideradas como tendo o mesmo grau de importância.

Com relação aos atributos de segundo nível "número de plantas em operação" e "número de plantas em construção/projeto", escolhidos para comparar as tecnologias no tocante aos riscos tecnológicos, foi consenso entre os técnicos especialistas em operação e projetos, que o número de plantas em operação seria um indicador mais forte do grau de confiabilidade do processo, devendo, portanto ter maior peso.

Obviamente, dependendo do processo de refino em questão, os pesos relativos aos diferentes atributos poderão variar e à medida que o procedimento for sendo utilizado, certamente surgirão novos aprimoramentos.

Em resumo, a definição dos atributos e os respectivos pesos foram atribuídos por especialistas das áreas de manutenção industrial, operação, projeto e meio ambiente com base em suas experiências no tipo de processo de refino que estava sendo analisado. Esse é um dos pontos nos quais podemos identificar um verdadeiro processo de conversão de conhecimento tácito em conhecimento explícito, o qual organizado de forma estruturada permite revisões periódicas e aprimoramento, se constituindo em um processo de aprendizagem organizacional conforme apontado por Senge (1990). 
A Tabela 5 apresenta os pesos dos atributos de segundo e terceiro níveis hierárquicos.

Tabela 5 - Pesos dos atributos de segundo e terceiro níveis $\left(\mathrm{H}_{2} \mathrm{~S}\right.$ : Sulfeto de Hidrogênio, $\mathrm{NH}_{3}$ : Amônia, $\mathrm{CO}_{2}$ : Dióxido de Carbono, $\mathrm{SO}_{\mathrm{x}}$ : Óxidos de Enxofre, $\mathrm{NO}_{\mathrm{x}}$ : Óxidos de Nitrogênio, COV: Compostos Orgânicos Voláteis).

\begin{tabular}{|c|c|c|c|}
\hline Atributos de segundo nível & Pesos & Atributos de terceiro nível & Pesos \\
\hline Dimensão dos equipamentos & 0,50 & $\begin{array}{c}\text { Fornos } \\
\text { Reatores } \\
\text { Torres e bandejas } \\
\text { Compressores } \\
\text { Filtros } \\
\text { Permutadores } \\
\text { Bombas } \\
\text { Vasos } \\
\text { Acionadores }\end{array}$ & $\begin{array}{l}0,11 \\
0,11 \\
0,11 \\
0,11 \\
0,11 \\
0,11 \\
0,11 \\
0,11 \\
0,11\end{array}$ \\
\hline Facilidades em geral & 0,50 & $\begin{array}{c}\text { Redundância de equipamentos } \\
\text { Disposição horizontal } \\
\text { Intercambiabilidade de peças } \\
\text { Sistemas liberáveis sem parada geral } \\
\text { Diversas entradas e saídas em } \\
\text { equipamentos } \\
\text { Emprego de materiais comuns } \\
\text { Sistemas na forma de skids } \\
\text { Dispositivos de fácil abertura } \\
\text { Área ocupada }\end{array}$ & $\begin{array}{l}0,11 \\
0,11 \\
0,11 \\
0,11 \\
0,11 \\
0,11 \\
0,11 \\
0,11 \\
0,11\end{array}$ \\
\hline Emissões líquidas & 0,33 & $\begin{array}{c}\text { Outros } \\
\mathrm{H}_{2} \mathrm{~S} \\
\mathrm{NH}_{3} \\
\text { Hidrocarbonetos }\end{array}$ & $\begin{array}{l}0,25 \\
0,25 \\
0,25 \\
0,25\end{array}$ \\
\hline Emissões gasosas & 0,33 & $\begin{array}{c}\mathrm{CO}_{2} \\
\mathrm{SO}_{\mathrm{x}} \\
\mathrm{NO}_{\mathrm{x}} \\
\mathrm{COV} \\
\text { Particulados }\end{array}$ & $\begin{array}{l}0,2 \\
0,2 \\
0,2 \\
0,2 \\
0,2\end{array}$ \\
\hline Descarte de sólidos & 0,33 & Taxa de descarte & 1,0 \\
\hline $\begin{array}{l}\text { Número de plantas em } \\
\text { construção/projeto }\end{array}$ & 0,20 & & \\
\hline $\begin{array}{l}\text { Número de plantas em } \\
\text { operação }\end{array}$ & 0,80 & & \\
\hline $\begin{array}{c}\text { Índice de intensidade } \\
\text { de energia }\end{array}$ & 1,0 & & \\
\hline Consumo de água & 1,0 & & \\
\hline
\end{tabular}




\subsubsection{Determinação da função de valor}

Com os pesos determinados, é possível calcular a função de valor global para diferentes tecnologias.

Tendo em vista que o teste da existência de interação entre os atributos associados à seleção de tecnologia apresentou resultado negativo, a utilização de uma função de valor aditiva é uma aproximação bastante adequada para o estabelecimento da função de valor global.

O modelo para a função de valor aditiva assume que existe um conjunto de funções de valor individuais, $\mathrm{V}_{(\mathrm{x} 1)}, \ldots . ., \mathrm{V}_{(\mathrm{xn})}$ para os $\mathrm{n}$ diferentes atributos e que cada uma dessas funções assume valores de 0 e 1 para o pior e o melhor resultado, respectivamente. A função de valor aditiva equivale à média ponderada dessas diferentes funções de valor, ou seja,

$$
\mathrm{V}_{\left(\mathrm{x}_{1}, \ldots \ldots, \mathrm{x}_{\mathrm{n}}\right)}=\mathrm{k}_{1} \mathrm{~V}_{\left(\mathrm{x}_{1}\right)}+\ldots .+\mathrm{k}_{\mathrm{n}} \mathrm{V}_{\left(\mathrm{x}_{\mathrm{n}}\right)}=\sum_{\mathrm{i}=1}^{\mathrm{n}} \mathrm{k}_{\mathrm{i}} \mathrm{V}_{\left(\mathrm{x}_{\mathrm{i}}\right)}
$$

Os pesos $k_{i}, \ldots, k_{n}$, em (2) são todos positivos e $\sum k_{i}=1$.

A função de valor aditiva assume valores de 0 a 1 para o pior e o melhor resultado possível, respectivamente.

Dessa forma, para cada tecnologia, a função de valor pode ser expressa por:

$\mathrm{V}(\mathrm{VPL}, \mathrm{RA}, \mathrm{FM}, \mathrm{RT}, \mathrm{CE}, \mathrm{CA})=\mathrm{K}_{\mathrm{VPL}}\left(\mathrm{V}_{\mathrm{VPL}}\right)+\mathrm{K}_{\mathrm{RA}}\left(\mathrm{V}_{\mathrm{RA}}\right)+\mathrm{K}_{\mathrm{FM}}\left(\mathrm{V}_{\mathrm{FM}}\right)+\mathrm{K}_{\mathrm{RT}}\left(\mathrm{V}_{\mathrm{RT}}\right)+\mathrm{K}_{\mathrm{CE}}\left(\mathrm{V}_{\mathrm{CE}}\right)+\mathrm{K}_{\mathrm{CA}}\left(\mathrm{V}_{\mathrm{CA}}\right)$

Em (3), tem-se:

$\mathrm{VPL}=$ Valor presente líquido,

RA $=$ Riscos ambientais,

FM $=$ Facilidade de manutenção,

$\mathrm{RT}=$ Riscos tecnológicos,

$\mathrm{CE}=$ Consumo de energia,

$\mathrm{A}=$ Consumo de água.

\subsection{Resultados}

A avaliação das tecnologias que serviram de base para a aplicação do modelo apresentou os resultados mostrados na Tabela 6 e Figura 1.

Tabela 6 - Função de valor multiatributo - Caso base.

\begin{tabular}{|c|c|c|c|c|c|c|c|}
\hline Pesos & 0,27 & 0,19 & 0,13 & 0,22 & 0,11 & 0,08 & $\begin{array}{c}\text { Função de Valor } \\
\text { Multiatributo }\end{array}$ \\
\hline Atributos & $\mathrm{V}_{\mathrm{VPL}}$ & $\mathrm{V}_{\mathrm{RA}}$ & $\mathrm{V}_{\mathrm{FM}}$ & $\mathrm{V}_{\mathrm{RT}}$ & $\mathrm{V}_{\mathrm{CE}}$ & $\mathrm{V}_{\mathrm{CA}}$ & \\
\hline Tec. $\mathrm{A}$ & 0,87 & 0,40 & 0,53 & 0,73 & 0,73 & 0,56 & 0,67 \\
\hline Tec. $\mathrm{B}$ & 0,00 & 0,18 & 0,73 & 0,20 & 1,00 & 1,00 & 0,36 \\
\hline Tec. C & 1,00 & 0,16 & 0,68 & 0,80 & 0,00 & 0,00 & 0,56 \\
\hline
\end{tabular}




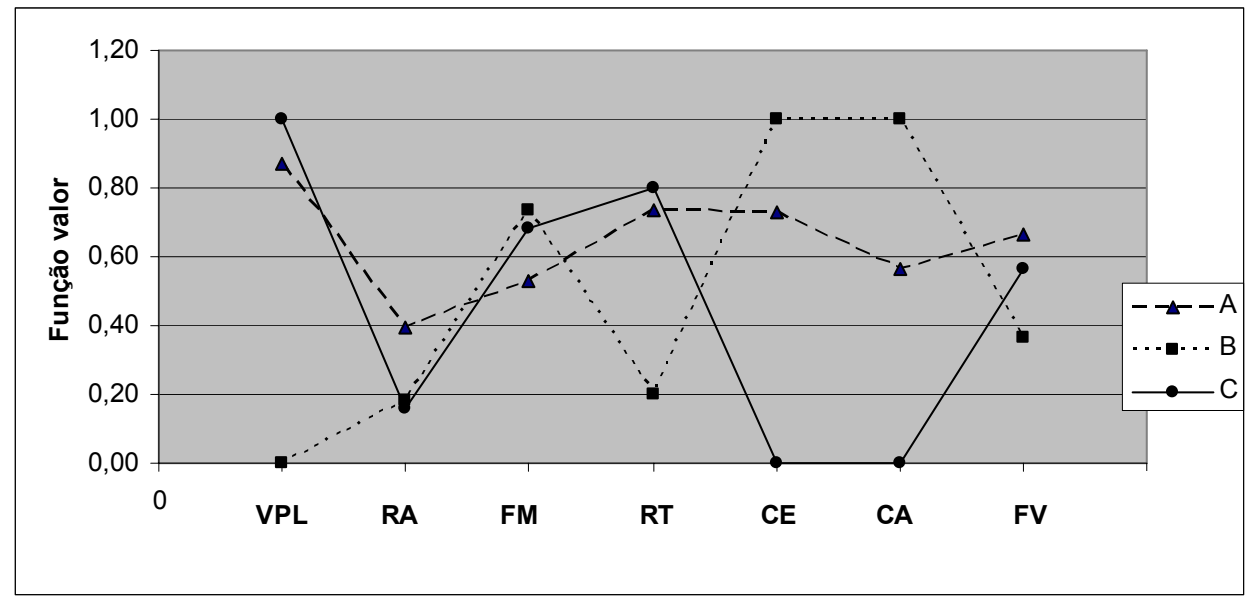

Figura 1 - Função de valor multiatributo - Caso base.

A tecnologia A foi a que apresentou maior função de valor global, seguida pela tecnologia C. A tecnologia B foi a que apresentou o pior resultado.

A tecnologia A, apesar de apresentar a funções de valor VPL, FM e RT inferiores à tecnologia $\mathrm{C}$, apresenta um desempenho acentuadamente melhor com relação às funções de valor RA, CE e CA, fazendo com que, no geral, apresente um melhor desempenho.

Um indicativo interessante sugerido no Gráfico 1 se refere à tecnologia $\mathrm{B}$, que de todas parece ser a mais otimizada em termos de utilização de recursos como água e energia, além da facilidade de manutenção, o que do ponto de vista operacional pode ser muito interessante. O fato de essa tecnologia apresentar a menor função de valor VPL, apesar do bom resultado com relação às outras funções, pode ser um indicativo de um ponto a ser questionado, junto ao licenciador da tecnologia, no sentido de concentrar esforços para o aumento daquela função de valor.

\subsubsection{Análise de Sensibilidade}

Os pesos atribuídos pelos especialistas aos diversos atributos serão função do cenário em questão e sempre haverá certo grau de subjetividade. Assim, é recomendável que seja feita uma análise de sensibilidade em relação aos pesos dos atributos, de forma a se avaliar o grau de robustez da escolha.

No caso base, os atributos "Valor presente líquido" e "Riscos tecnológicos" foram priorizados, ou seja, a esses atributos foram atribuídos maiores pesos. Entretanto, em um cenário diferente, os pesos alocados aos atributos poderiam variar de forma considerável, levando a uma escolha diferente. Assim, por exemplo, em um ambiente em que o insumo água seja escasso, o peso do atributo "Consumo de água" poderia ser maior. Da mesma forma, em um contexto em que a localização da planta seja tal que dificulte o acesso de grandes equipamentos para montagem, o atributo "Facilidade de manutenção" poderia ser priorizado.

Assim, como forma de se avaliar o grau de robustez da escolha, os pesos alocados aos atributos de primeiro nível foram variados, alternando-se dois a dois com relação ao caso 
base. A Tabela 7 apresenta os casos alternativos que foram considerados na análise de sensibilidade e a Tabela 8 os respectivos resultados das funções de valor multiatributo.

Tabela 7 - Pesos alternativos dos atributos de primeiro nível

( $\mathrm{V}_{\mathrm{VPL}}$ : Função de valor "Valor presente líquido", $\mathrm{V}_{\mathrm{RA}}$ : Função de valor "Riscos ambientais",

$\mathrm{V}_{\mathrm{FM}}$ : Função de valor "Facilidade manutenção", $\mathrm{V}_{\mathrm{RT}}$ : Função de valor "Riscos tecnológicos",

$\mathrm{V}_{\mathrm{CE}}$ : Função de valor "Consumo de energia", $\mathrm{V}_{\mathrm{CA}}$ : Função de valor "Consumo de água").

\begin{tabular}{|c|c|c|c|c|c|c|}
\hline & $\mathrm{V}_{\mathrm{VPL}}$ & $\mathrm{V}_{\mathrm{RA}}$ & $\mathrm{V}_{\mathrm{FM}}$ & $\mathrm{V}_{\mathrm{RT}}$ & $\mathrm{V}_{\mathrm{CE}}$ & $\mathrm{V}_{\mathrm{CA}}$ \\
\hline Caso Base & 0,27 & 0,19 & 0,13 & 0,22 & 0,11 & 0,08 \\
\hline Caso $\mathrm{X}_{1}$ & 0,19 & 0,27 & 0,13 & 0,22 & 0,11 & 0,08 \\
\hline Caso $\mathrm{X}_{2}$ & 0,13 & 0,19 & 0,27 & 0,22 & 0,11 & 0,08 \\
\hline Caso $\mathrm{X}_{3}$ & 0,22 & 0,19 & 0,13 & 0,27 & 0,11 & 0,08 \\
\hline Caso $\mathrm{X}_{4}$ & 0,11 & 0,19 & 0,13 & 0,22 & 0,27 & 0,08 \\
\hline Caso $\mathrm{X}_{5}$ & 0,08 & 0,19 & 0,13 & 0,22 & 0,11 & 0,27 \\
\hline Caso $\mathrm{X}_{6}$ & 0,27 & 0,13 & 0,19 & 0,22 & 0,11 & 0,08 \\
\hline Caso $\mathrm{X}_{7}$ & 0,27 & 0,22 & 0,13 & 0,19 & 0,11 & 0,08 \\
\hline Caso $\mathrm{X}_{8}$ & 0,27 & 0,11 & 0,13 & 0,22 & 0,19 & 0,08 \\
\hline Caso $\mathrm{X}_{9}$ & 0,27 & 0,08 & 0,13 & 0,22 & 0,11 & 0,19 \\
\hline Caso $\mathrm{X}_{10}$ & 0,27 & 0,19 & 0,22 & 0,13 & 0,11 & 0,08 \\
\hline Caso $\mathrm{X}_{11}$ & 0,27 & 0,19 & 0,22 & 0,11 & 0,13 & 0,08 \\
\hline Caso $\mathrm{X}_{12}$ & 0,27 & 0,19 & 0,22 & 0,11 & 0,08 & 0,13 \\
\hline Caso $\mathrm{X}_{13}$ & 0,27 & 0,19 & 0,13 & 0,11 & 0,22 & 0,08 \\
\hline Caso $\mathrm{X}_{14}$ & 0,27 & 0,19 & 0,13 & 0,11 & 0,08 & 0,22 \\
\hline Caso $\mathrm{X}_{15}$ & 0,27 & 0,19 & 0,13 & 0,22 & 0,08 & 0,11 \\
\hline
\end{tabular}

Tabela 8 - Função de valor multiatributo - Casos alternativos.

\begin{tabular}{|c|c|c|c|}
\hline & Tec. $\mathrm{A}$ & Tec. $\mathrm{B}$ & Tec. $\mathrm{C}$ \\
\hline Caso $\mathrm{X}_{1}$ & 0,63 & 0,38 & 0,50 \\
\hline Caso $\mathrm{X}_{2}$ & 0,62 & 0,47 & 0,52 \\
\hline Caso $\mathrm{X}_{3}$ & 0,66 & 0,37 & 0,56 \\
\hline Caso $\mathrm{X}_{4}$ & 0,64 & 0,52 & 0,41 \\
\hline Caso $\mathrm{X}_{5}$ & 0,61 & 0,55 & 0,38 \\
\hline Caso $\mathrm{X}_{6}$ & 0,67 & 0,40 & 0,60 \\
\hline Caso $\mathrm{X}_{7}$ & 0,66 & 0,36 & 0,55 \\
\hline Caso $\mathrm{X}_{8}$ & 0,69 & 0,43 & 0,55 \\
\hline Caso $\mathrm{X}_{9}$ & 0,68 & 0,45 & 0,55 \\
\hline Caso $\mathrm{X}_{10}$ & 0,65 & 0,41 & 0,55 \\
\hline Caso $\mathrm{X}_{11}$ & 0,65 & 0,43 & 0,54 \\
\hline Caso $\mathrm{X}_{12}$ & 0,64 & 0,43 & 0,54 \\
\hline Caso $\mathrm{X}_{13}$ & 0,67 & 0,45 & 0,48 \\
\hline Caso $\mathrm{X}_{14}$ & 0,64 & 0,45 & 0,48 \\
\hline Caso $\mathrm{X}_{15}$ & 0,66 & 0,36 & 0,57 \\
\hline
\end{tabular}


A Figura 2 apresenta os resultados da função de valor multiatributo para cada uma das tecnologias em função de diferentes pesos dos atributos de primeiro nível.

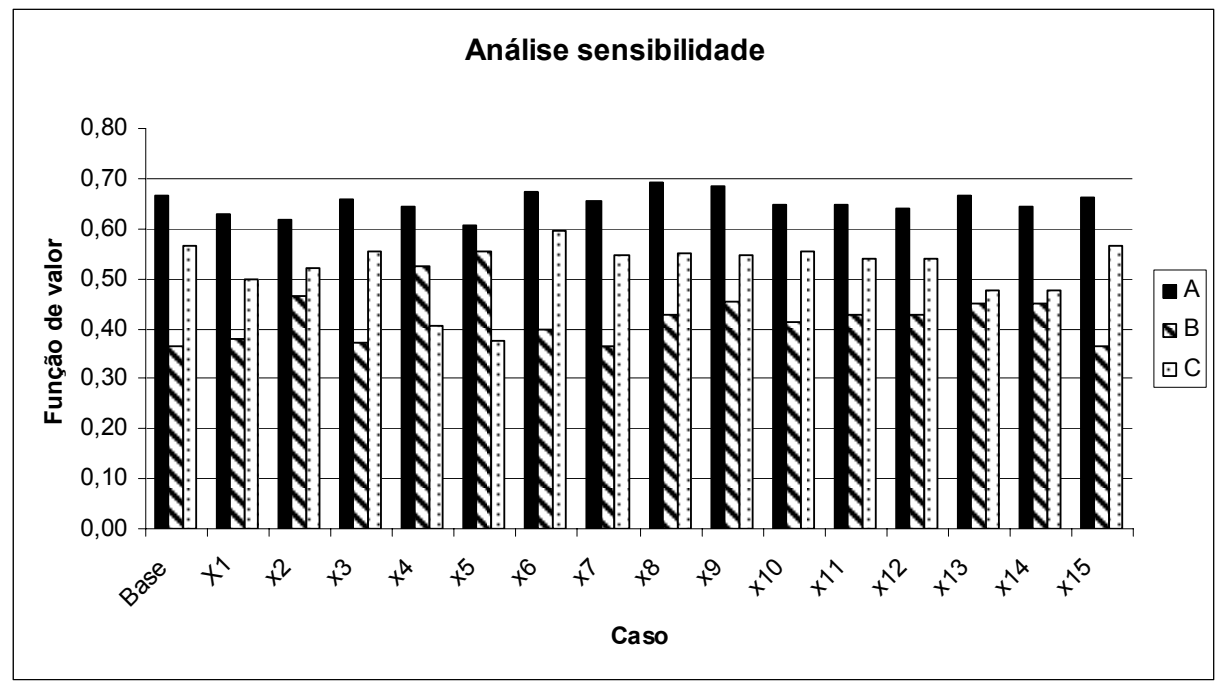

Figura 2 - Função de valor multiatributo - Casos alternativos.

A análise da Figura 2 permite concluir que, mesmo com mudanças nos pesos alocados aos atributos de primeiro nível, a tecnologia A ainda se mantém como a melhor opção, demonstrando ser uma escolha robusta, capaz de atender a diferentes contextos.

Em $87 \%$ dos casos analisados, a tecnologia C demonstrou ser a segunda melhor opção, porém, em $13 \%$ dos casos, a tecnologia B foi a segunda melhor opção, sinalizando que, em função de alguma especificidade do cenário, a escolha pode variar.

Desta forma, a análise de sensibilidade com relação aos pesos dos atributos é sempre recomendável para se avaliar o grau de robustez da escolha.

\section{Conclusão}

O estudo de caso apresentado neste artigo mostrou como os métodos analíticos de apoio à decisão podem ser utilizados como instrumentos para gestão do conhecimento em determinadas áreas tecnológicas. A partir da aplicação da função de valor multiatributo para seleção de tecnologias de processos de refino de petróleo, procurou-se demonstrar como a informação adequadamente estruturada permite captar o conhecimento tácito dos especialistas, convertendo-o em conhecimento explícito, além de aprimorar a qualidade e conferir agilidade ao processo decisório.

A função de valor multiatributo foi o método que, por suas características, se mostrou o mais adequado à abordagem do problema; no entanto, é conveniente ressaltar que a escolha do método será sempre em função das características do problema.

Os métodos analíticos de apoio à decisão muitas vezes apresentam grau de complexidade que dificultam a sua utilização como metodologia rotineira nas empresas, uma vez que buscam 
reproduzir o comportamento dos indivíduos em seu processo de escolha. A maneira de contornar o problema é o desenvolvimento de sistemas previamente modelados, específicos para cada aplicação; sistemas que possam conferir a agilidade necessária ao processo decisório e que se constituam em ferramentas de conversão de conhecimento tácito em explícito, tendo em vista que esses sistemas serão construídos a partir do conhecimento teórico e vivência prática de técnicos especialistas. Cabe ressaltar, porém, que é fundamental que os usuários conheçam as premissas dos métodos analíticos utilizados e que as modelagens estejam continuamente sujeitas a reavaliações, revalidações ou atualizações. Somente dessa forma elas se constituirão em instrumentos de aprendizagem organizacional.

A utilização de um método multicritério de forma interativa apresenta a vantagem adicional de permitir que, a cada resultado, sejam feitas novas avaliações e eventuais correções. A adoção de tal método foi possível devido às características do problema permitir uma representação adequada em termos de atributos quantitativos, e a árvore de critérios ter sido dimensionada de forma satisfatória, representando as preferências e culturas dos diversos atores do processo decisório.

Apesar de alguns trabalhos empíricos demonstrarem que nem sempre os indivíduos tomam decisões coerentes com os axiomas propostos pelas teorias da decisão, é consenso entre diversos autores que é de se esperar que tomadores de decisões, relacionadas aos interesses públicos ou de terceiros, adotem estratégias que possam ser justificadas com base em preceitos lógicos e explícitos; daí a contribuição dos métodos multicritério no contexto das organizações.

A modelagem do problema foi desenvolvida com base na experiência dos técnicos em processos de seleção de tecnologia de refino de petróleo. Procurou-se adotar atributos que pudessem caracterizar os processos de refino em geral. À medida que a metodologia for continuamente aplicada, a modelagem dos atributos poderá ser aprimorada, no sentido de representar especificamente cada uma das tecnologias de processos, de forma a melhor registrar o conhecimento relativo àquelas tecnologias.

\section{Agradecimentos}

Os autores agradecem à Petrobras S.A, através de sua Área de Negócio AbastecimentoRefino, por propiciarem os meios indispensáveis para realização deste trabalho. Os autores agradecem ainda a dois revisores anônimos, cujas recomendações contribuíram para introduzir melhoramentos substanciais na primeira versão deste artigo.

\section{Referências Bibliográficas}

(1) Belton, V.; Ackermann, F. \& Sheperd, I. (1997). Integrated support for problem structuring through an alternative evaluation using COPE and V.I.S.A. Journal of Multi-Criteria Decision Analysis, 6, 115-130.

(2) Belton, V. \& Stewart, T. (2002). Multiple Criteria Decision Analysis: An Integrated Approach. Kluwer Academic Publishers, Boston.

(3) Bentham, J. (1789). Introduction to the Principles of Morals and Legislation. Primeira edição impressa em 1780 e publicada em 1789. Londres.

(4) Bouyssou, D. (1990). Building Criteria: A prerequisite for MCDA. In: Readings in Multiple Criteria Decision Aid. Springer, New York. 
(5) Brown, R. (2005). Rational Choice and Judgment Decision Analysis for the Decider. John Wiley \& Sons, Hoboken.

(6) Campos, M.L. de (2005). Um Processo de Estruturação do Conhecimento Organizacional. Tese submetida ao Programa de Pós-graduação em Engenharia de Produção da Universidade Federal de Santa Catarina para a obtenção do grau de Doutor em Engenharia. UFSC, Florianópolis.

(7) Clemen, R. \& Reilly, T. (2001). Making Hard Decisions with Decision Tools. Pacific Grove, Duxbury.

(8) Dyer, J.S. (2005). MAUT - Multiattribute Utility Theory. In: Multiple Criteria Decision Analysis: State of the Art Surveys [edited by Figueira, J.; Greco, S. \& Ehrgott, M.], Springer, New York, 265-295.

(9) Ensslin, L.; Montibeller Neto, G. \& Noronha, S.M.D. (2001). Apoio à Decisão: Metodologias para Estruturação de Problemas e Avaliação Multicritério de Alternativas. 1. ed. Insular, Florianópolis.

(10) Feyzioğlu, O. \& Büyüközkan, G. (2007). Fuzzy Multi-criteria Evaluation of Knowledge Management Tools. In: 10th Joint Conference on Information Sciences 2007. Society for Mathematics of Uncertainty, Salt Lake City.

(11) Figueira, J.; Greco, S. \& Ehrgott, M. (eds.) (2005). Multiple Criteria Decision Analysis State of the Art Surveys. Springer, New York.

(12) Fishburn, P.C. (1970). Utility Theory for Decision-Making. Wiley, New York.

(13) Gomes, L.F.A.M.; Gomes, C.F.S.; Almeida, A.T. (2006). Tomada de Decisão Gerencial - Enfoque Multicritério. Atlas, São Paulo.

(14) Gomes, L.F.A.M. \& Santos, J.L.C.F. (2008). Knowledge management and the analytical methods for decision support. Rio's International Journal on Sciences of Industrial and Systems Engineering and Management, 2, 1-12. Disponível em $<$ http://www.rij.eng.uerj.br/scientific/2008/>.

(15) Kain, J.-H.; Kärrman, E. \& Söderberg, H. (2007) Multi-criteria Decision Aids for Sustainable Water Management. Engineering Sustainability, 160(2), 87-93.

(16) Keeney, R.L. \& Raiffa, H. (1976). Decisions with Multiple Objectives- Preferences and Value Tradeoffs. Wiley, New York.

(17) Montibeller Neto, G.; Belton, V.; Ackermann, F. \& Ensslin, L. (2008). Reasoning maps for decision aid: an integrated approach for problem-structuring and multi-criteria evaluation. Journal of the Operational Research Society, 59, 575-589.

(18) Morey, D.; Maybury, M. \& Thuraisingham, B. (2002). Knowledge Management: Classic and Contemporary Works. MIT Press, Cambridge.

(19) Nonaka, I. \& Takeuchi, H. (1995). The Knowledge-creating Company: How Japanese Companies Create the Dynamics of Innovation. Oxford University Press, New York.

(20) Phillips, L.D. (1984). A theory of requisite decision models. Acta Psychologica, 52, $29-48$.

(21) Phillips, L.D. (1990). Requisite decision modelling for technological projects. In: Social Decision Methodology for Technological Projects [edited by Vlek, C. \& Cvetkovich, G.], Kluwer Academic Publishers, Dordrecht, 95-110. 
(22) Rauscher, H.M.; Schmoldt, D.L. \& Vacik, H. (2007). Information and Knowledge Management in Support of Sustainable Forestry: A Review. In: Sustainable Forestry: from Monitoring and Modelling to Knowledge Management and Policy Science, 439-460. Disponível em <http://treesearch.fs.fed.us/pubs>.

(23) Refining Processes Handbook (2008). Hydrocarbon processing. Gulf Publishing Company, Houston.

(24) Rosenhead, J. \& Mingers, J. (eds.) (2008). Rational Analysis for a Problematic World revisited. $2^{\text {nd }}$ ed. John Wiley \& Sons, Chichester.

(25) Santos, J.L.C.F. (2003). O Papel do Suporte Analítico à Tomada de Decisão na Gestão do Conhecimento Organizacional. Dissertação de Mestrado Profissionalizante em Administração, Faculdades Ibmec/RJ, Rio de Janeiro.

(26) Senge, P. (1990). The Fifth Discipline: The Art and Practice of the Learning Organization. Doubleday Currency, New York.

(27) Souza, F.M.C. de (2002). Decisões Racionais em Situações de Incerteza. Ed. Universitária UFPE, Recife.

(28) Trinkaus, H.L. (2006). Multi Criteria Knowledge Management. In: EURO XXI in Iceland $-21^{\text {st }}$ European Conference in Operational Research. EURO, Reykjavick.

(29) Von Neumann, J. \& Morgernstern, O. (1947). Theory of Games and Economic Behavior. $2^{\text {nd }}$ ed. Princeton University Press, Princeton.

(30) Wallenius, J.; Dyer, J.S.; Fishburn, P.; Steuer, R.E.; Zionts, S. \& Deb, K. (2008). Multiple Criteria Decision Making, Multiattribute Utility Theory: Recent Accomplishments and What Lies Ahead. Management Sciences, 54(7), 1336-1349.

(31) Zeleny, M. (2005). Human Systems Management Integrating knowledge, management and systems. World Scientific Printers, Singapore. 